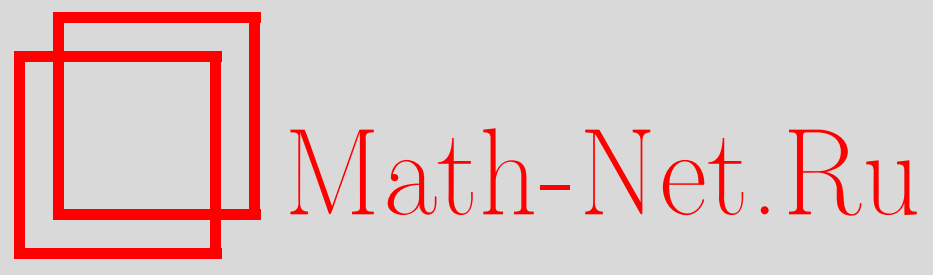

А. Г. Гринь, О минимальном условии слабой зависимости в центральной предельной теореме для стационарных последовательностей, Теория вероятн. и ее примен., 2002, том 47, выпуск 3, 554-558

DOI: https://doi.org/10.4213/tvp3694

Использование Общероссийского математического портала MathNet.Ru подразумевает, что вы прочитали и согласны с пользовательским соглашением

http://www . mathnet.ru/rus/agreement

Параметры загрузки:

IP: 52.23 .180 .231

26 апреля 2023 г., $07: 26: 01$

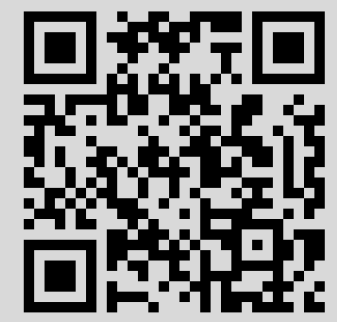


(C) $2002 \mathrm{r}$.

ГРИНЬ А. Г. ${ }^{*}$

\title{
О МИНИМАЛЬНОМ УСЛОВИИ СЛАБОЙ ЗАВИСИМОСТИ В ЦЕНТРАЛЬНОЙ ПРЕДЕЛЬНОЙ ТЕОРЕМЕ ДЛЯ СТАЦИОНАРНЫХ ПОСЛЕДОВАТЕЛЬНОСТЕЙ ${ }^{1)}$
}

\begin{abstract}
Предложено минимальное в некотором смысле условие слабой зависимости для стационарных последовательностей, обеспечивающее выполнение центральной предельной теоремы.
\end{abstract}

Ключевые слова и фразы: стационарные последовательности, условия слабой зависимости, центральная предельная теорема.

Пусть $\left\{\xi_{n}\right\}$ - стационарная в узком смысле последовательность, и пусть $S_{n}=$ $\sum_{k=1}^{n} \xi_{k}$. Будем писать $\xi \stackrel{\mathrm{d}}{=} \eta, \xi_{n} \stackrel{\mathrm{d}}{\rightarrow} \eta$ и $\xi_{n} \stackrel{\mathrm{d}}{\sim} \eta_{n}$ в случаях, когда, соответственно, распределения $\xi$ и $\eta$ совпадают, $\left\{\xi_{n}\right\}$ сходится к $\eta$ по распределению и когда последовательности $\left\{\xi_{n}\right\}$ и $\left\{\eta_{n}\right\}$ слабо эквивалентны (см., например, [1, §28.1]). Слабая эквивалентность равносильна поточечной сходимости разности характеристических функций величин $\xi_{n}$ и $\eta_{n}$ к нулю при $n \rightarrow \infty[1$, с. 393]. Обозначим через $\mathscr{N}(0,1)$ случайную величину, имеющую стандартное нормальное распределение.

Если $\mathbf{E} \xi_{n}^{2}<\infty, n=1,2, \ldots, \sigma_{n}^{2}=\mathbf{D} S_{n} \rightarrow \infty$ и

$$
\sigma_{n}^{-1}\left(S_{n}-\mathbf{E} S_{n}\right) \stackrel{\mathrm{d}}{\longrightarrow} \mathscr{N}(0,1), \quad n \rightarrow \infty,
$$

то будем говорить, что к последовательности $\left\{\xi_{n}\right\}$ применима центральная предельная теорема.

Следуя [2], назовем $\left\{b_{n}, n=1,2, \ldots\right\}$ правильно меняющейся последовательностью порядка $\rho$, если $b_{[x]}, x>0$, является правильно меняющейся функцией порядка $\rho$, где $[x]$ - целая часть $x$.

Предельные теоремы для сумм зависимых случайных величин, как правило, доказываются с определенными заранее условиями слабой зависимости (регулярности) такими, как условия сильного перемешивания, равномерно сильного перемешивания, полной регулярности ( $\rho$-перемешивания) и т.п. (см., например, [3]). В связи с этим возникает вопрос - насколько выполнение той или иной теоремы обусловлено данным условием регулярности, возможно ли (и в каких границах) ослабление этого условия?

В настоящей заметке предлагается минимальное в некотором смысле условие слабой зависимости, при котором справедлива центральная предельная теорема.

Естественно, центральная предельная теорема может иметь место и при сколь угодно зависимых слагаемых, что показывают, скажем, примеры такого типа: $\xi_{n}=$ $\mathscr{N}(0,1), n=1,2, \ldots$, или $\xi_{n}=\eta_{n}+\mathscr{N}(0,1), n=1,2, \ldots$, где $\left\{\eta_{n}\right\}-$ последовательность независимых и не зависяших от $\mathscr{N}(0,1)$ одинаково распределенных величин с конечной дисперсией. Легко видеть, что в этих случаях $\sigma_{n} \sim n, n \rightarrow \infty$.

Вместе с тем в предельных теоремах для последовательностей с сильным перемешиванием, равномерно сильным перемешиванием, полной регулярностью нормальное предельное распределение может иметь место лишь в случае, когда масштабная нормировка осуществляется правильно меняющимися последовательностями порядка $\frac{1}{2}$ (см., например, [3, теорема 18.1.1]). Предположение о том, что $\sigma_{n}$ является правильно меняющейся последовательностью порядка $\frac{1}{2}$, является, по существу, некоторым условием слабой зависимости, касающимся лишь моментов порядка не выше второго (см.

* Омский государственный университет, пр. Мира, 55a, 644077 Омск, Россия.

1) Работа выполнена при поддержке Российского фонда фундаментальных исследований (грант 99-01-01130) и программы интегращии науки и высшей школы (грант 586). 
лемму 1), и это предположение в дальнейшем позволит нам «отсеять» последовательности, не обладаюшие слабой зависимостью, подобные приводившимся выше.

Через $\widehat{\xi}_{1}, \ldots, \widehat{\xi}_{n}$ будем обозначать независимые случайные величины такие, что $\widehat{\xi}_{k} \stackrel{\mathrm{d}}{=} \xi_{k}, k=1,2, \ldots, n$.

Будем говорить, что последовательность $\left\{\xi_{n}\right\}$ удовлетворяет условию (R), если при любом действительном $t$ и при любой последовательности натуральных чисел $m=m(n)$

$$
\mathbf{E} \exp \left\{i t \sigma_{n+m}^{-1} S_{n+m}\right\}-\mathbf{E} \exp \left\{i t \sigma_{n+m}^{-1} S_{n}\right\} \mathbf{E} \exp \left\{i t \sigma_{n+m}^{-1} S_{m}\right\} \longrightarrow 0,
$$

$n \rightarrow \infty$ (для краткости будем говорить, что соотношение $(\mathrm{R})$ выполняется при $n+$ $m \rightarrow \infty)$. В соответствии с определением слабой эквивалентности условие (R) можно записать так:

$$
\frac{S_{n+m}}{\sigma_{n+m}} \stackrel{\mathrm{d}}{\sim} \frac{\widehat{S}_{n}}{\sigma_{n+m}}+\frac{\widehat{S}_{m}}{\sigma_{n+m}}, \quad n+m \rightarrow \infty .
$$

Лемма 1. Дисперсия $\sigma_{n}^{2}$ является правильно меняюшейся последовательностью порядка 1 (а $\sigma_{n}$ - правильно меняющейся последовательностью порядка $\left.\frac{1}{2}\right)$ тогда и только тогда, когда выполнено «условие (R) в широком смысле»:

$$
\sigma_{n+m}^{2} \sim \sigma_{n}^{2}+\sigma_{m}^{2}, \quad n+m \rightarrow \infty .
$$

Теорема 1. Пусть $\left\{\xi_{n}, n=1,2, \ldots\right\}-$ стачионарная последовательность, $u$ пусть $\mathbf{E} \xi_{n}^{2}<\infty, \mathbf{E} \xi_{n}=0, n=1,2, \ldots$ Для того чтобы $\kappa$ последовательности $\left\{\xi_{n}\right\}$ бьла применима чентральная предельная теорема $и \sigma_{n}$ являлась правильно меняющейся Функиией порядка $\frac{1}{2}$, необходимо и достаточно, чтобы выполнялось условие (R) и последовательность $\left\{\sigma_{n}^{-2} S_{n}^{2}\right\}$ была равномерно интегрируема.

Доказательства леммы и теоремы приводятся ниже.

3 а м е ч а н и е 1 . Теорему 1 можно интерпретировать так: условие (R) является минимальным условием слабой зависимости, при котором справедлива центральная предельная теорема с правильно меняющейся порядка 1 дисперсией.

3 а м е ч а н и е 2. Из условий сильного перемешивания, равномерно сильного перемешивания, $\rho$-перемешивания следует условие (R). Это доказывается стандартным методом секционирования (см., например, [3, теорема 18.1.1]). Наоборот, из условия (R) ни одно из названных выше условий, вообще говоря, не следует, более того, условие $(\mathrm{R})$ может выполняться для последовательностей со сколь угодно сильной зависимостью «прошлого» и «будушего». Простейший пример такой последовательности: $\xi_{n}=\eta_{n}+(-1)^{n} \zeta, n=1,2, \ldots$, где $\eta_{n}-$ последовательность независимых одинаково распределенных величин, а $\zeta-$ случайная величина с симметричным распределением, не зависящая от $\left\{\eta_{n}\right\}, \mathbf{E} \eta_{n}^{2}<\infty, \mathbf{E} \zeta^{2}<\infty$. Тогда $\sigma_{n}^{2} \sim n \mathbf{D} \eta_{n}$ и

$$
\sigma_{n}^{-1} S_{n}=\sigma_{n}^{-1}\left(\sum_{k=1}^{n} \eta_{k}+\frac{1}{2}\left((-1)^{n}-1\right) \zeta\right) \stackrel{\mathrm{d}}{\sim} \sigma_{n}^{-1} \sum_{k=1}^{n} \eta_{k},
$$

откуда легко следуют и условие (R), и применимость центральной предельной теоремы к последовательности $\left\{\xi_{n}\right\}$. В то же время ясно, что эта последовательность не удовлетворяет, вообще говоря, ни одному из общепринятых условий слабой зависимости. Попросту говоря, условие $(\mathrm{R})$ - это слабая зависимость сумм, а не слагаемых.

3 а м е ч а н и е 3 . Если последовательность $\left\{\xi_{n}\right\}$ удовлетворяет условию $\rho$-перемешивания и $\sigma_{n} \rightarrow \infty, n \rightarrow \infty$, то $\sigma_{n}$ является правильно меняюшейся последовательностью порядка $\frac{1}{2}([3$, замечание 18.2.2]). Из теоремы 1 и замечания 2 следует теперь, что для последовательности с $\rho$-перемешиванием центральная предельная теорема имеет место тогда и только тогда, когда последовательность $\left\{\sigma_{n}^{-2} S_{n}^{2}\right\}$ равномерно интегрируема. Этот факт неоднократно доказывался многими авторами (см., например, [4]).

Д ока 3 а т ель с т в о л е м мы 1. Мы будем доказывать, что $\sigma_{n}^{2}$ является правильно меняющейся последовательностью порядка 1 , для чего достаточно проверить справедливость следующих соотношений (см., например, [5]):

$$
\sigma_{n}^{-2} \sigma_{n+1}^{2} \rightarrow 1, \quad \sigma_{n}^{-2} \sigma_{k n}^{2} \rightarrow k, \quad n \rightarrow \infty
$$


при любом натуральном $k$ и $\sigma_{[(\lambda+\mu) n]}^{2} \sim \sigma_{[\lambda n]}^{2}+\sigma_{[\mu n]}^{2}, n \rightarrow \infty$, где $\lambda>0, \mu>0$. Легко видеть, что эти соотношения вытекают из (2), так что если выполнено (2), то $\sigma_{n}$ является правильно меняющейся последовательностью порядка $\frac{1}{2}$.

Наоборот, пусть $\sigma_{n}$ является правильно меняюшейся последовательностью порядка $\frac{1}{2}$. Тогда $\sigma_{n}^{2}=n h(n)$, где $h(x)-$ медленно меняюшаяся функция. Для функции $h(x)$ справедливы следующие утверждения:

$$
\lim _{n \rightarrow \infty} \sup _{\varepsilon \leqslant \lambda \leqslant 1}\left|\frac{h(\lambda n)}{h(n)}-1\right|=0, \quad \varepsilon>0,
$$

[2, теорема 1.1],

[3, лемма 18.2.4]. Имеем

$$
\lim _{\varepsilon \rightarrow 0} \lim _{n \rightarrow \infty} \sup _{0<\lambda<\varepsilon} \frac{\lambda h(\lambda n)}{h(n)}=0
$$

$$
\frac{\sigma_{n}^{2}+\sigma_{m}^{2}}{\sigma_{n+m}^{2}}=\frac{n h(n)}{(n+m) h(n+m)}+\frac{m h(m)}{(n+m) h(n+m)}=I_{1}+I_{2} .
$$

Легко видеть, что существует последовательность $\varepsilon_{n} \rightarrow 0, n \rightarrow \infty$, такая, что соотношения (3) и (4) выполняются при $\varepsilon=\varepsilon_{n}$. Пусть для определенности $n \geqslant m$. Если $m \geqslant n \varepsilon_{n}$, то в силу (3)

$$
I_{1}+I_{2}=\frac{n}{n+m}\left(1+o_{n}(1)\right)+\frac{m}{n+m}\left(1+o_{n}(1)\right)=1+o_{n}(1)
$$

Если же $m<n \varepsilon_{n}$, то в силу (4) $I_{2}=o_{n}(1)$, так что снова $I_{1}+I_{2}=1+o_{n}(1)$. Лемма доказана.

Д ок аз а т ел ь с т в о т е о ре м ы 1. Необходимость. Пусть к последовательности $\left\{\xi_{n}\right\}$ применима центральная предельная теорема, т.е. при любом $t \in \mathbf{R}$

$$
\mathbf{E} \exp \left\{i t \sigma_{n}^{-1} S_{n}\right\} \longrightarrow \exp \left\{-\frac{t^{2}}{2}\right\}, \quad n \rightarrow \infty,
$$

и $\sigma_{n}$ является правильно меняющейся функцией порядка $\frac{1}{2}$. В этих предположениях равномерная интегрируемость последовательности $\left\{\sigma_{n}^{-2} S_{n}^{2}\right\}$ доказана, например, в [3, c. 425$]$.

Пусть $t \in \mathbf{R}$ и $m=m(n)$. Обозначим

$$
\Delta(n)=\left|\mathbf{E} \exp \left\{i t \sigma_{n+m}^{-1} S_{n+m}\right\}-\mathbf{E} \exp \left\{i t \sigma_{n+m}^{-1} S_{n}\right\} \mathbf{E} \exp \left\{i t \sigma_{n+m}^{-1} S_{m}\right\}\right|
$$

Поскольку $\sigma_{n}^{2}$ - правильно меняюшаяся последовательность порядка 1 , то $\sigma_{n+m}^{2} \sim$ $\sigma_{n}^{2}+\sigma_{m}^{2}, n \rightarrow \infty$, в силу леммы 1 , так что для любой последовательности натуральных чисел $\left\{n_{1}\right\}$ существуют $0 \leqslant c \leqslant 1$ и подпоследовательность $\left\{n_{2}\right\} \subseteq\left\{n_{1}\right\}$ такие, что при $n \rightarrow \infty$

$$
\sigma_{n_{2}+m_{2}}^{-2} \sigma_{n_{2}}^{2} \rightarrow c, \quad \sigma_{n_{2}+m_{2}}^{-2} \sigma_{m_{2}}^{2} \rightarrow 1-c,
$$

где $m_{2}=m\left(n_{2}\right)$. Если $c=0(c=1)$, то при $n \rightarrow \infty$

$$
\sigma_{n_{2}+m_{2}}^{-1} S_{n_{2}} \rightarrow 0 \quad\left(\sigma_{n_{2}+m_{2}}^{-1} S_{m_{2}} \rightarrow 0\right)
$$

по вероятности, следовательно, $\Delta\left(n_{2}\right) \rightarrow 0, n \rightarrow \infty$. Если же $0<c<1$, то в силу (15)

$$
\begin{aligned}
\mathbf{E} \exp \left\{i t \sigma_{n_{2}+m_{2}}^{-1} S_{n_{2}+m_{2}}\right\} & \sim \exp \left\{-\frac{t^{2}}{2}\right\}=\exp \left\{-\frac{c t^{2}}{2}\right\} \exp \left\{-\frac{(1-c) t^{2}}{2}\right\} \\
& \sim \mathbf{E} \exp \left\{i t \sigma_{n_{2}+m_{2}}^{-1} S_{n_{2}}\right\} \mathbf{E} \exp \left\{i t \sigma_{n_{2}+m_{2}}^{-1} S_{m_{2}}\right\}, \quad n \rightarrow \infty
\end{aligned}
$$

т.е. снова $\Delta\left(n_{2}\right) \rightarrow 0, n \rightarrow \infty$.

Таким образом, доказано, что из любой последовательности $\left\{\Delta\left(n_{1}\right)\right\}$ можно выделить сходящуюся к нулю подпоследовательность. Это означает, что $\Delta(n) \rightarrow 0$, $n \rightarrow \infty$, т.е. выполнено условие $(\mathrm{R})$. 
Достаточность. Пусть выполнено условие (R) и последовательность $\left\{\sigma_{n}^{-2} S_{n}^{2}\right\}$ равномерно интегрируема. В силу известной теоремы Прохорова (см., например, [6]) последовательность $\left\{\sigma_{n}^{-1} S_{n}\right\}$ является относительно компактной, так что из любой последовательности натуральных чисел можно выбрать подпоследовательность $\left\{n_{1}\right\}$, $n_{1}=n_{1}(n)$, такую, что при $n \rightarrow \infty$

$$
\sigma_{n_{1}}^{-1} S_{n_{1}} \stackrel{\mathrm{d}}{\longrightarrow} \xi, \quad \sigma_{m_{1}}^{-1} S_{m_{1}} \stackrel{\mathrm{d}}{\longrightarrow} \eta, \quad \sigma_{n_{1}+m_{1}}^{-1} S_{n_{1}+m_{1}} \stackrel{\mathrm{d}}{\longrightarrow} \zeta,
$$

где $m_{1}=m\left(n_{1}\right)$, а $\xi, \eta$ и $\zeta-$ случайные величины. При этом поскольку последовательность $\left\{\sigma_{n}^{-2} S_{n}^{2}\right\}$ равномерно интегрируема, то

$$
\mathbf{E} \xi^{2}=\lim _{n \rightarrow \infty} \mathbf{E}\left(\sigma_{n_{1}}^{-1} S_{n_{1}}\right)^{2}=1, \quad \mathbf{E} \eta^{2}=1, \quad \mathbf{E} \zeta^{2}=1
$$

[6, теорема 5.4]. Далее, из ограниченных последовательностей $\alpha_{n_{1}}=\sigma_{n_{1}} / \sqrt{\sigma_{n_{1}}^{2}+\sigma_{m_{1}}^{2}}$, $\beta_{n_{1}}=\sigma_{m_{1}} / \sqrt{\sigma_{n_{1}}^{2}+\sigma_{m_{1}}^{2}}$ выберем подпоследовательности $\left\{\alpha_{n_{2}}\right\}$ и $\left\{\beta_{n_{2}}\right\}$ такие, что

$$
\alpha_{n_{2}} \rightarrow \alpha, \quad \beta_{n_{2}} \rightarrow \beta, \quad n \rightarrow \infty, \quad \alpha^{2}+\beta^{2}=1 .
$$

Тогда

$$
\frac{\widehat{S}_{n_{2}}+\widehat{S}_{m_{2}}}{\sqrt{\sigma_{n_{2}}^{2}+\sigma_{m_{2}}^{2}}}=\alpha_{n_{2}} \sigma_{n_{2}}^{-1} \widehat{S}_{n_{2}}+\beta_{n_{2}} \sigma_{m_{2}}^{-1} \widehat{S}_{m_{2}} \stackrel{\mathrm{d}}{\longrightarrow} \alpha \widehat{\xi}+\beta \widehat{\eta}, \quad n \rightarrow \infty .
$$

Понятно, что $\alpha \widehat{\xi}+\beta \widehat{\eta}$ имеет невырожденное распределение.

Далее, в силу соотношений (1), (6) и (7)

$$
\sigma_{n_{2}+m_{2}}^{-1}\left(\widehat{S}_{n_{2}}+\widehat{S}_{m_{2}}\right) \stackrel{\mathrm{d}}{\longrightarrow} \zeta, \quad n \rightarrow \infty
$$

где $\zeta$ имеет невырожденное распределение. По теореме о сходимости типов $[1$, c. 216$]$ из (9) и (10) вытекает $\sigma_{n_{2}+m_{2}}^{-1} \sqrt{\sigma_{n_{2}}^{2}+\sigma_{m_{2}}^{2}} \longrightarrow C, 0<C<\infty$. Отсюда следует, что вместе с последовательностями $\left\{\sigma_{n_{2}}^{-2} S_{n_{2}}^{2}\right\}$ и $\left\{\sigma_{m_{2}}^{-2} S_{m_{2}}^{2}\right\}$ равномерно интегрируемой является последовательность $\left\{\sigma_{n_{2}+m_{2}}^{-2}\left(\widehat{S}_{n_{2}}^{2}+\widehat{S}_{m_{2}}^{2}\right)\right\}$, и из (10) получаем теперь $\delta_{n_{2}}=\sigma_{n_{2}+m_{2}}^{-2}\left(\sigma_{n_{2}}^{2}+\sigma_{m_{2}}^{2}\right) \longrightarrow \mathbf{E} \zeta^{2}=1, n \rightarrow \infty$. Таким образом, мы показали, что для всякой последовательности натуральных чисел найдется подпоследовательность $\left\{n_{2}\right\}$ такая, что $\delta_{n_{2}} \rightarrow 1, n \rightarrow \infty$. Это означает, что $\delta_{n} \rightarrow 1, n \rightarrow \infty$, т.е. выполнено (2). По лемме $1 \sigma_{n}$ является правильно меняющейся последовательностью порядка $\frac{1}{2}$.

Пусть $\sigma_{n}$ является правильно меняющейся последовательностью порядка $\frac{1}{2}$ и выполнено условие (R). Представим произвольное натуральное $n$ в виде $n=k m+r$, $m<n, 0 \leqslant r<m, k=[n / m]$. Тогда

$$
S_{n}=\sum_{j=1}^{k} \sum_{i=(j-1) m+1}^{j m} \xi_{i}+\sum_{i=k m+1}^{k m+r} \xi_{i}
$$

Из свойства (2) легко выводится, что

$$
\sup _{n \geqslant 1} \max _{1 \leqslant r \leqslant m} \sigma_{r}^{2} \sigma_{m}^{-2}<\infty
$$

так что

$$
\left(\sqrt{k} \sigma_{m}\right)^{-1} \sum_{i=k m+1}^{k m+r} \xi_{i} \longrightarrow 0
$$

по вероятности, а последовательность $k=k(n) \rightarrow \infty$ можно подобрать растущей столь медленно, что

$$
\sigma_{n}^{2} \sim k \sigma_{m}^{2}
$$

Из условия (R) (уменьшив, если нужно, скорость стремления $k(n)$ к бесконечности) и соотношений (13) и (14) получаем

$$
\sigma_{n}^{-1} \sum_{j=1}^{k} \sum_{i=(j-1) m+1}^{j m} \xi_{i} \stackrel{\mathrm{d}}{\sim}\left(\sqrt{k} \sigma_{m}\right)^{-1} \sum_{j=1}^{k} X_{j}, \quad n \rightarrow \infty,
$$


где $X_{j} \stackrel{\mathrm{d}}{=} S_{m}$ и $X_{1}, \ldots, X_{k}$ - независимы. Для того чтобы к последовательности серий $\left\{\left(\sqrt{k} \sigma_{m}\right)^{-1} X_{j}, j=1, \ldots, k, n=1,2, \ldots\right\}$ независимых случайных величин была применима центральная предельная теорема, достаточно, чтобы выполнялось условие Линдеберга: при любом $\varepsilon>0$

$$
L_{n}(\varepsilon)=\frac{1}{k \sigma_{m}^{2}} \sum_{j=1}^{k} \mathbf{E}\left\{X_{j}^{2},\left|X_{j}\right|>\varepsilon \sqrt{k} \sigma_{m}\right\} \longrightarrow 0, \quad n \rightarrow \infty .
$$

Используя определение величин $X_{j}$ и равномерную интегрируемость последовательности $\left\{\sigma_{m}^{-2} S_{m}^{2}\right\}$, получаем $L_{n}(\varepsilon)=\sigma_{m}^{-2} \mathbf{E}\left\{S_{m}^{2},\left|S_{m}\right|>\varepsilon \sqrt{k} \sigma_{m}\right\} \longrightarrow 0, n \rightarrow \infty$, что вместе с (11) и (15) дает

$$
\sigma_{n}^{-1} S_{n} \stackrel{d}{\sim}\left(\sqrt{k} \sigma_{m}\right)^{-1} \sum_{j=1}^{k} X_{j} \stackrel{d}{\longrightarrow} \mathscr{N}(0,1), \quad n \rightarrow \infty .
$$

Теорема доказана.

\section{СПИСОК ЛИТЕРАТУРЫ}

1. Лоэв М. Теория вероятностей. М.: ИЛ, 1962,719 с.

2. Сенета $E$. Правильно меняющиеся функции. М.: Наука, 1985, $141 \mathrm{c.}$

3. Ибрагимов И. А., Линник Ю. В. Независимые и стационарно связанные величины. М.: Наука, 1965, 524 с.

4. Denker $M$. Uniform integrability and the central limit theorem for strongly mixing processes. - Progr. Probab. Statist., 1986, v. 11, p. 269-274.

5. Гринь $A . Г$. Области притяжения для последовательностей с перемешиванием. Сиб. матем. журн., 1990, т. 31, № 1, с. 53-63.

6. Биллингсли П. Сходимость вероятностных мер. М.: Наука, 1977, 351 с.

Поступила в редакцию 27.VII. 2000

(C) $2002 \Gamma$.

ИВЧЕНКО Г. И., МЕДВЕДЕВ Ю. И.

\section{МЕТОД В. Л. ГОНЧАРОВА И ЕГО РАЗВИТИЕ В АНАЛИЗЕ РАЗЛИЧНЫХ МОДЕЛЕЙ СЛУЧАЙНЫХ ПОДСТАНОВОК}

Демонстрируется эффективность метода производящих функций, впервые использованного В.Л. Гончаровым при анализе асимптотических свойств случайных подстановок, в задаче исследования совместных распределений максимальных длин циклов случайной подстановки в модели Эванса. Выводится явный вид соответствующей многомерной плотности Гончарова и ее обобщения.

Ключевые слова и фразы: случайные подстановки и их структура, случайные многочлены над конечными полями, производящие функции, предельные теоремы.

* Московский государственный институт электроники и математики, Б. Трехсвятительский пер., 3/12, 109028 Москва, Россия. 